\title{
Sensitive Colorimetric Visualization of Perfluorinated Compounds Using Poly(ethylene glycol) and Perfluorinated Thiols Modified Gold Nanoparticles
}

\author{
Hongyun Niu, ${ }^{\dagger}$ Saihua Wang, ${ }^{\dagger, \dagger}$ Zhen Zhou, ${ }^{\dagger}$ Yurong Ma, ${ }^{\S}$ Xunfeng Ma, ${ }^{\ddagger}$ and Yaqi Cai* ${ }^{\dagger}$ \\ ${ }^{\dagger}$ State Key Laboratory of Environmental Chemistry and Ecotoxicology, Research Center for Eco-Environmental Sciences, \\ Chinese Academy of Sciences, Beijing 100085, China \\ ${ }^{\ddagger}$ School of Urban and Environmental Science, Northeast Normal University, Changchun, Jilin Province China \\ ${ }^{\S}$ School of Environmental Science and Engineering, Yancheng Institute of Technology, Yancheng, Jiangsu Province China
}

ABSTRACT: In this work, we have developed a novel sensing strategy employing mixed poly(ethylene glycol)-terminated (PEG-thiols) and perfluoroalkyl-terminated (F-thiols) alkanethiols modified gold nanoparticles (Au@PEG-F NPs) as a probe to detect perfluorinated compounds (PFCs) from water samples. PEG-thiols with high density and long carbon chains make the Au NPs probe well-dispersed in solution and stable even in high concentration of salt solution; F-thiols provide specific fluorous-fluorous interactions to PFCs, which results in adsorption of PFCs on Au@PEG-F NPs. The adsorbed PFCs cause the aggregation of Au@PEG-F NPs probes and thus induce the insolubility of probes and precipitation directly from reaction

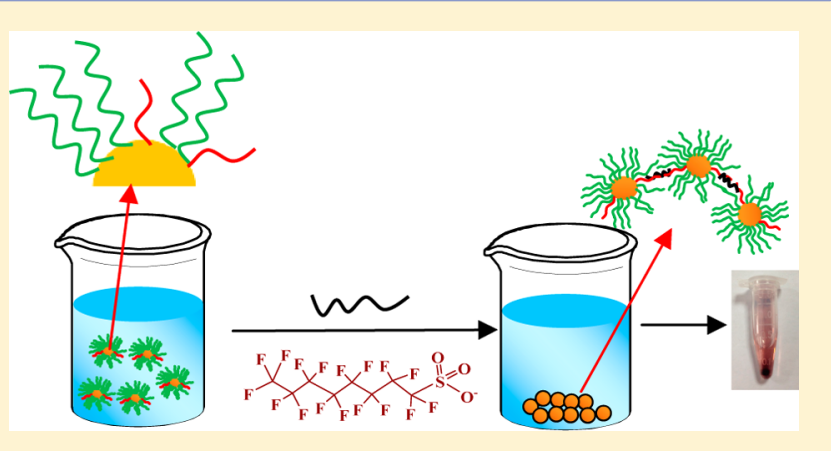
solution due to the superhydrophobicity of perfluorocarbon monolayers, leading to color and absorbance response of the assay to PFCs. The preparation of the Au@PEG-F NPs probe is very simple, and the colorimetric assay based on this mechanism for the detection of PFCs is selective and convenient. Combined with UV-vis spectrophotometry, the assay demonstrates good sensitivities to PFCs with wide linear range. In the designed concentration range, the response of the colorimetric assay to longchain PFCs (perfluoroalkyl chain $\geq 7$ ) is discerned even as the concentration of these PFCs is as low as $10 \mu \mathrm{g} \mathrm{L}{ }^{-1}$. This low-cost and sensitive assay shows great potential to measure total PFCs in water samples. To the best of our knowledge, this is the first application of the specific fluorous-fluorous interactions and Au NPs based probes for colorimetric recognition for PFCs.

$\mathrm{P}$ erfluorinated compounds (PFCs) are a specialty class of chemicals employed for a variety of applications, including lubricants, paints, cosmetics, and fire-fighting foams. They are highly stable, bioaccumulative, and resistant to degrade in the environments and have been regarded as a new class of emerging organic pollutants. PFCs have been detected in the atmosphere, water, soil, and wildlife all over the world, even in Antarctic and Arctic areas. ${ }^{1-5}$ Most PFCs lack chromophores and are nonvolatile; derivatization techniques coupled with spectrofluorometric detector, ${ }^{6}$ high-performance liquid chromatography with fluorescence detection (HPLC-FLD), ${ }^{7}$ gas chromatography with electron capture detection (GC-ECD), and mass spectrometry with electron impact ionization (GC-EI-MS $)^{8,9}$ were employed for the determination of PFCs. However, these methods have limited utility for the detection of PFCs in environmental matrixes due to the complex procedure. ${ }^{5}$ Ion chromatography coupled with conductivity detection and an electrophoretic method to measure PFCs are also reported; but the limits of detection are general in the milligram per liter level. ${ }^{10,11}$ These days, the routine and robust analytical technique for PFCs is liquid chromatography/tandem mass spectrometry (LC/MS/MS), which still is too expensive for common application in environmental monitoring. Thus, the development of simple, easy-to-operate, inexpensive, and sensitive assays for PFCs is in high demand.

Over the past decade, gold nanoparticles (Au NPs) based colorimetric assays have become an important research topic and been applied to monitor (bio)chemical substances. The concentration change of targets can be easily transformed into color changes, which is observed by the naked eye alone; hence, no sophisticated instruments are required. The color change of $\mathrm{Au}$ NPs colloid is highly sensitive to the size, shape, capping agents, and medium refractive index, as well as the aggregation state of Au NPs. ${ }^{12}$ Combined with UV-vis spectrophotometry or fluorescence resonance energy transfer techniques, Au NPs based colorimetric assays for DNA, proteins, ions, and organic molecules (such as cysteine, melamine, cocaine, ATP, pyrophosphate ion, glucose, and dopamine) have been developed. ${ }^{12-26}$ However, the establishment of colorimetric assays for PFCs has not been reported yet. Actually, the applications of colorimetric assays based on Au NPs for environmental pollutants are scarce,

Received: October 20, 2013

Accepted: March 31, 2014

Published: March 31, 2014 
and only very few colorimetric assays have been designed for TNT, ${ }^{27,28}$ organophosphorus and carbamate pesticides, ${ }^{29}$ and phthalates. $^{30}$

Selective isolation of fluorinated targets from convoluted mixtures has become increasingly popular using liquid-liquid separations, solid-liquid separations (such as fluorous solidphase extraction), and fluorous chromatography. ${ }^{31-37}$ Most of these types of separations rely on a fluorous silica solid phase coupled with an organic solvent. On the basis of the highly specific fluoruos-fluorous $(\mathrm{F}-\mathrm{F})$ interactions, fluorous molecules are retained by fluorous silica gel while nonfluorous molecules are not. ${ }^{32,34}$ Fluorous-tagged analytes such as triphenylphosphine oxide, ${ }^{33,34}$ fluoroalkyl-substituted phenyl bromides, ${ }^{35}$ oligonucleotides, ${ }^{36}$ and peptides ${ }^{37}$ have been successfully separated from nonfluorous analytes by using fluorinated adsorbent or fluorinated monolithic columns. However, there is no application of fluorinated Au NPs in adsorption, separation, or detection of fluorous analytes by far.

In the present study, Au NPs based colorimetric assays for PFCs were established. The surfaces of Au NPs were functionalized with mixed self-assembled monolayers of alkanethiolates terminated with poly(ethylene glycol) (PEG-thiols) and alkanethiolates terminated with perfluoroalkyl (F-thiols) (Au@PEG-F NPs). The PEG-thiols increased the dispersibility of Au NPs in water, while the F-thiols were capable of binding PFCs by providing unique $\mathrm{F}-\mathrm{F}$ interactions to PFCs. The adsorption of PFCs on Au@PEG-F NPs probes surface increased the hydrophobicity of the probes and led to the precipitation of Au@PEG-F NPs out of solution. The color of Au@PEG-F colloids faded clearly as PFCs were added into the solution, and the absorbance decreased linearly with PFCs concentration. The comparison study showed that the nonperfluorinated compounds did not interfere with the detection of PFCs.

\section{EXPERIMENTAL SECTION}

Materials and Instrumentation. Chloroauric acid tetrahydrate $\left(\mathrm{HAuCl}_{4} \cdot 4 \mathrm{H}_{2} \mathrm{O}\right)$, sodium borohydride $\left(\mathrm{NaBH}_{4}\right)$, and ethanol were obtained from Sinopharm Chemical Reagent Company, Ltd. (Beijing, China). Alkanethiol terminated with poly(ethylene glycol) $\mathrm{HS}\left(\mathrm{CH}_{2}\right)_{11}-\left(\mathrm{OCH}_{2} \mathrm{CH}_{2}\right)_{n}-\mathrm{OH}(n=6)$ and alkanethiol terminated with perfluoroalkyl $\mathrm{HS}\left(\mathrm{CH}_{2}\right)_{11}-$ $\mathrm{O}-\left(\mathrm{CH}_{2}\right)_{2}-\left(\mathrm{CF}_{2}\right)_{5}-\mathrm{CF}_{3}$ were from ProChimia, Poland. Perfluorobutanesulfonate (PFBS), perfluorohexanesulfonate (PFHxS), perfluoroheptanoic acid (PFHpA), perfluorooctanoic acid (PFOA), perfluorononanoic acid (PFNA), perfluorotridecanoic acid (PFTrDA), perfluorotetradecanoic acid (PFTeDA), perfluorohexadecanoic acid (PFHxDA), and perfluorooctadecanoic acid (PFODA) were purchased from Alfa Aesar; perfluorooctanesulfonate (PFOS) was obtained from SigmaAldrich. All chemicals were used as received without further purification. Ultrapure water was prepared using a Milli-Q SP reagent water system (Millipore, Bedford, MA, U.S.A.).

UV-vis spectra were recorded using a Beckman DU 800 nucleic acid/protein analyzer (Rosemead, CA). X-ray photoelectron spectroscopy (XPS) measurements were conducted by applying a Thermo Scientific ESCA-Lab-200i-XL spectrometer (Waltham, MA) with monochromatic $\mathrm{Al} \mathrm{K \alpha}$ radiation (1486.6 eV). The size and morphology of Au@PEG-F NPs was observed with a Hitachi H-7500 transmission electron microscope (TEM, Tokyo, Japan). The amount of Au NPs in colloid after addition of PFCs was determined using inductively coupled plasma mass spectrometry (ICPMS) (7500A, Agilent).
The $\zeta$-potential of Au@PEG-F NPs at different pHs and hydrodynamic radius, Rh (nm), of Au@PEG-F NPs in the presence and absence of PFCs were measured using Zetasizer Nano series, (Malvern, United Kingdom).

Synthesis of PEG- and F-thiols Mixed Capped AuNPs (Au@PEG-F) and Sensing PFCs. The AuNPs were prepared by reducing $\mathrm{HAuCl}_{4}$ with $\mathrm{NaBH}_{4}$ in the presence of PEG thiols as stabilizers. In brief, $1.872 \mathrm{mg}$ of PEG-thiols was added to $40 \mathrm{~mL}$ of $\mathrm{HAuCl}_{4}$ ethanol solution $\left(5 \times 10^{-4} \mathrm{M}\right)$; the terminal concentration of PEG-thiols was $0.1 \mathrm{mM}$. After mixing, $1 \mathrm{~mL}$ of freshly prepared $0.1 \mathrm{M}$ of $\mathrm{NaBH}_{4}$ solution was added into the mixture and the color of solution turned to deep red immediately. The reaction solution was stirred for $30 \mathrm{~min}$. Next, $0.275 \mathrm{mg}$ of F-thiols was added into the PEG-capped Au NPs dispersion; the terminal concentration of F-thiols was $0.0125 \mathrm{mM}$. The mixture stood overnight. After that, the ethanol solvent was evaporated to about $1 \mathrm{~mL}$ under $\mathrm{N}_{2}$ stream followed with redispersing the PEG- and F-thiols mixed capped $\mathrm{Au}$ NPs with $40 \mathrm{~mL}$ of purified water. Finally, the Au@PEG-F NPs suspension was centrifuged at $13000 \mathrm{rpm}$ to remove the possible precipitated Au@PEG-F NPs. The developed reagent was stored at room temperature until use.

The colorimetric sensing of PFCs using the Au NPs probes was performed by mixing different amounts of PFCs solution with the as-synthesized Au@PEG-F NPs colloids at a volume ratio of $1: 1$. Since the concentrations of organic pollutants in the environment usually are expressed as mass per liter, the mass concentrations were used for PFCs in this study. After incubation for $0.5 \mathrm{~h}$ at room temperature, the precipitated $\mathrm{Au} @$ PEG-F NPs were removed from the reaction solution through centrifugation at $13000 \mathrm{rpm}$. The supernatants are used for absorbance measurement at a wavelength of $520 \mathrm{~nm}$.

\section{RESULTS AND DISCUSSION}

Colorimetric Sensor for PFCs. The amount of Au@PEG-F NPs sensors in the suspension was $0.25 \mathrm{mM}$ (representing the concentration of $\mathrm{Au}^{3+}$ in the initial reaction solution to synthesize Au NPs). After addition of different concentrations of PFCs, the reaction solution was incubated for $30 \mathrm{~min}$. The color of the Au@PEG-F NPs suspension faded gradually with the increase of PFCs (PFOS) concentration, and a small amount of Au@PEG-F NPs precipitated from reaction solutions (Figure 1A). The adsorption spectrum for each sample is shown in Figure 1B. The results exhibited that only the surface plasmon resonance (SPR) peak at about $520 \mathrm{~nm}$ presented in the spectra regardless of the concentration of PFOS, and the absorbance of reaction solution decreased linearly with the increase of PFOS concentration (Figure 1C). The shift in the spectra accompanying the binding of PFOS to $\mathrm{Au} @$ PEG-F NPs did not occur regardless of the amount of PFOS present.

To investigate the mechanism of the assay, TEM images of as-prepared Au@PEG-F NPs and those in the presence of PFOS (ranged in $0.1-1000 \mu \mathrm{g} \mathrm{L}^{-1}$ ) were taken. After reaction, the Au@PEG-F NPs colloid suspensions were centrifuged at $13000 \mathrm{rpm}$ and the supernatants were collected for TEM observations. As a result, the as-synthesized Au@PEG-F NPs were well-dispersed and about 2-4 $\mathrm{nm}$ in diameter (Figure 2A). With the addition of $0.1 \mu \mathrm{g} \mathrm{L}^{-1}$ of PFOS, only two or three Au NPs were drawn closer to one another, and the number of these "aggregated Au NPs" was low (Figure 2B); as PFOS concentration was enhanced to $0.5 \mu \mathrm{g} \mathrm{L}^{-1}$, the number of aggregated $\mathrm{Au}$ NPs increased clearly and more $\mathrm{Au}$ NPs were 


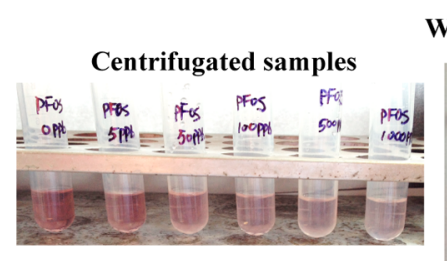

Without centrifugation
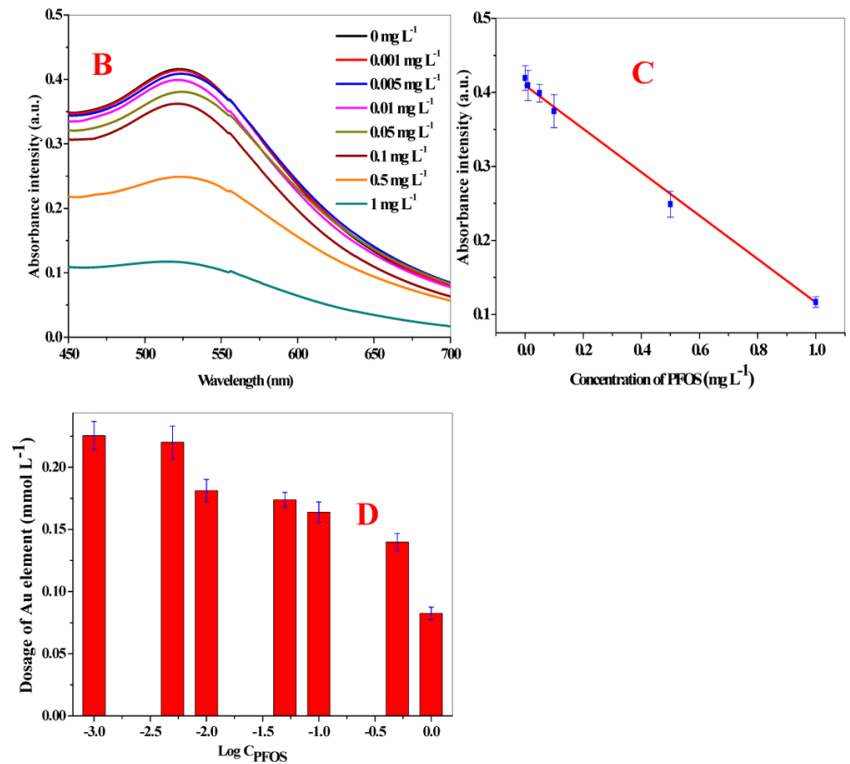

Figure 1. (A) Photographs of centrifuged Au@PEG-F NPs suspension containing different amounts of PFOS; from left to right: 0, 5, 50, 100, 500, and $1000 \mu \mathrm{g} \mathrm{L}^{-1}$, and Au@PEG-F NPs suspension before and after reaction with PFOS without centrifugation. (B) Absorbance of $\mathrm{Au} @$ PEG-F NPs suspension at $\mathrm{pH} 5.5$ in response to different concentrations of PFOS. (C) The plot of absorbance against $C_{\mathrm{PFOS}}$ for PFOS analysis. (D) $\mathrm{T}$ corresponding amount of $\mathrm{Au}$ atom in supernatants after reaction.

drawn close (Figure 2C). However, the association of a small amount of Au NPs would be insufficient to generate the color change and shift of the SPR peak in UV-vis spectra. With the further increase of PFOS concentration, the degree and number of "aggregated Au NPs" decreased gradually and tiny deposited $\mathrm{Au}$ NPs were seen in the bottle of the cuvette after centrifugation (Figure 2D). When the concentration of PFOS was higher than $10 \mu \mathrm{g} \mathrm{L} \mathrm{L}^{-1}$, aggregation of nanoparticles disappeared in the supernatant of reaction solution (Figure 2E). The precipitates were also examined using TEM and found to be composed of heavily aggregated Au@PEG-F NPs (Figure 2F). We measured the average hydrodynamic radii (Rhs) of controlled Au@PEG-F NPs and those in the supernatant after reaction with $500 \mu \mathrm{g} \mathrm{L}^{-1}$ of PFOS. Figure 3 exhibits that the
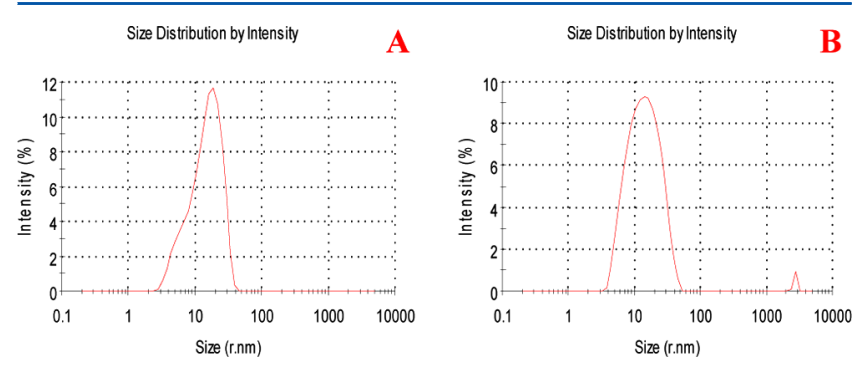

Figure 3. Hydrodynamic radius $(\mathrm{Rh})$ distributions of as-prepared $\mathrm{Au} @$ PEG-F NPs (A) and Au@PEG-F NPs after reaction with $500 \mu \mathrm{g} \mathrm{L}^{-1}$ PFOS (B). Both of the solutions are centrifuged, and the supernatants are used to determine $\mathrm{Rh}$ of probes.

Rhs of as-prepared Au@PEG-F NPs are 15.8 nm and identical with those of the probers that remained in the supernatant after reaction with PFOS $(15.7 \mathrm{~nm})$.

The next experiment was conducted to determine the amount of $\mathrm{Au}$ element in the supernatant of the suspension after reaction with PFOS. The results showed that the amounts of $\mathrm{Au}$ element decreased with the increase of PFOS concentration (Figure 1D). This phenomenon was rather consistent with the trend of absorbance at $520 \mathrm{~nm}$ in UV-vis spectra (Figure 1B), suggesting that the decreased absorbance of the reaction solution was due to the precipitation of $\mathrm{Au} @$ PEG-F NPs.

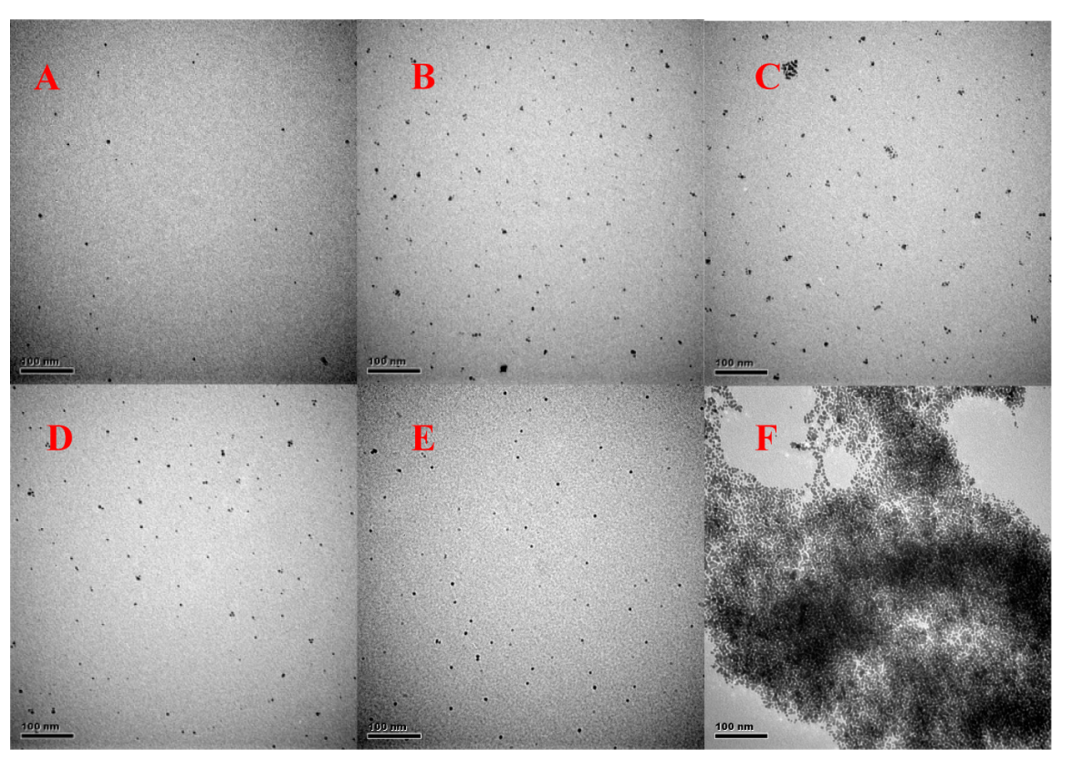

Figure 2. TEM images of as-prepared Au@PEG-F NPs (A) and Au@PEG-F NPs in the supernatants of reaction solution with the addition of 0.1 (B), 0.5 (C), 1.0 (D), and $10 \mu \mathrm{g} \mathrm{L}^{-1}$ (E) of PFOS, and heavily aggregated Au@PEG-F NPs in precipitates (F); the scale bar is $100 \mathrm{~nm}$. 
In a preliminary study, we optimized the ratios of PEG-thiols to F-thiols on the surface of Au NPs probes. We found that gold nanoparticles merely modified with PEG-thiols (Au@PEG NPs) were deep red in color and exhibited an SPR peak at about $515 \mathrm{~nm}$. As the ratios of PEG/F were 20:1 and 10:1 (representing the ratio of PEG-thiols and F-thiols added in reaction solution), the $\mathrm{Au}$ NPs colloids were deep wine red with the SPR peaks located at about $518 \mathrm{~nm}$. As the PEG/F thiols ratio decreased to $8: 1$, the nanoparticles were also deep wine red, but the SPR peak shifted to $520 \mathrm{~nm}$; at the same time small amounts of Au@PEG-F (8:1) deposited from water solution. If the PEG/F ratio decreased to 6:1, plenty of Au NPs deposited from polar solvents (methanol, ethanol, water). After removal of the precipitated Au NPs through centrifugation, the $\mathrm{Au} @$ PEG-F (6:1) NPs suspension was pale red, while the SPR peak was still located at about $520 \mathrm{~nm}$. We collected Au@PEG-F (8:1) NPs and Au@PEG-F (6:1) NPs well-dispersed in solution by evaporating the reaction solution under $\mathrm{N}_{2}$ stream, subsequently washing with ethanol and water several times to determine the element contents of Au@PEG-F (8:1) and Au@ PEG-F (6:1) using XPS. Consequently, the ratios of PEG/F thiols were about $8.7 / 1$ on the surface of the two Au NPs (Table 1), indicating that the minimum PEG/F thiols ratio on

Table 1. Element Composition and Contents on the Surface of Au NPs Modified with Different Ratios of PEG- and F-thiols

\begin{tabular}{cccc} 
element & $\begin{array}{c}\text { Au@PEG NPs } \\
(\%)\end{array}$ & $\begin{array}{c}\text { Au@PEG-F (8:1) NPs } \\
(\%)\end{array}$ & $\begin{array}{c}\text { Au@PEG-F (6:1) NPs } \\
(\%)\end{array}$ \\
$\mathrm{Au}$ & 1.06 & 1.09 & 0.98 \\
$\mathrm{~S}$ & 1.74 & 1.88 & 2.01 \\
$\mathrm{C}$ & 74.1 & 72.6 & 70.4 \\
$\mathrm{O}$ & 23.1 & 20.2 & 22.5 \\
$\mathrm{~F}$ & & 4.23 & 4.11 \\
\hline
\end{tabular}

Au NPs sensor was 8.7. The fluorocarbon monolayers are superhydrophobic, and the fluorinated alkyl thiolate [such as perfluorodecanethiol (F8) and perfluorooctanethiol (F6)] modified $\mathrm{Au}$ NPs are insoluble in common hydrocarbon media and only well-dispersed in fluorinated solvents. ${ }^{38-40}$ Therefore, further increase of the perfluorinated groups on $\mathrm{Au} @$ PEG-F NPs will enhance the hydrophobicity of Au NPs sensor, which makes the sensor insoluble in polar solvents and finally deposits from reaction solution. That is the reason for the same characteristic SPR peak in the spectra of Au@PEG-F (8:1) and Au@PEG-F (6:1).

All the above-mentioned results indicated that the added PFCs would adsorb on the surface of Au@PEG-F NPs through F-thiols, which might cause the aggregation of Au NPs. With the increase of PFCs in reaction solution, more Au NPs were drawn closer, resulting in the increase of the Au NPs probes' hydrophobicity. Since the perfluorocarbon monolayers are superhydrophobic and readily precipitate from water solution, the aggregated $\mathrm{Au}$ NPs would precipitate directly from reaction solution as the concentration of PFCs was high enough, leading to color and absorbance response of the assay to PFCs. The possible mechanism of the assay is shown in Figure 4.

Effects of Solution pH and Electrolytes. The stability of $\mathrm{Au} @$ PEG-F NPs in solution at different pHs was investigated using UV-vis spectroscopy. In the $\mathrm{pH}$ range of 2.5-6.5, the absorbance reduced gradually and a sharp decrease occurred at $\mathrm{pH} 7.5$, after which the intensities increased with $\mathrm{pH}$ values (Figure 5A). The change of extinction coefficient with solution

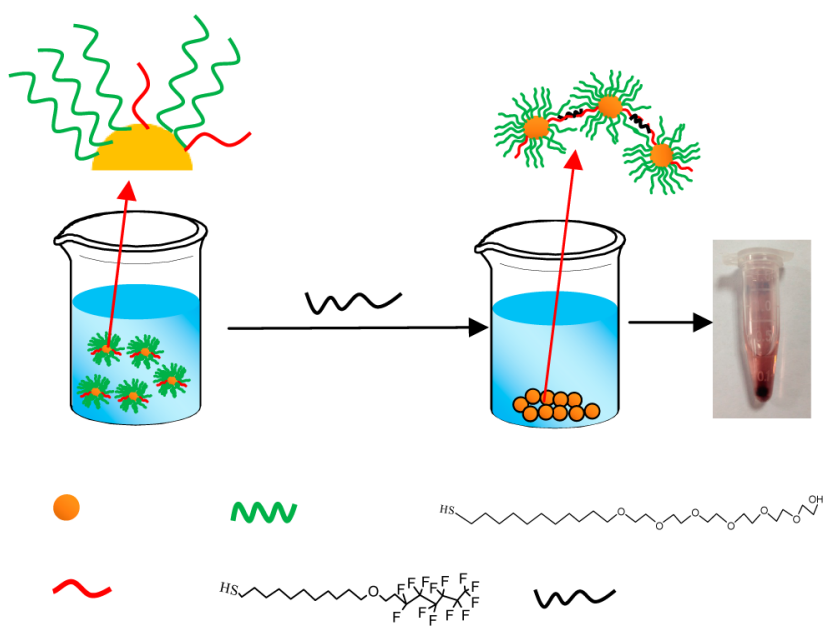

Figure 4. Mechanistic assay for colorimetric detection of PFCs with perfluorinated and PEG thiols modified Au NPs.

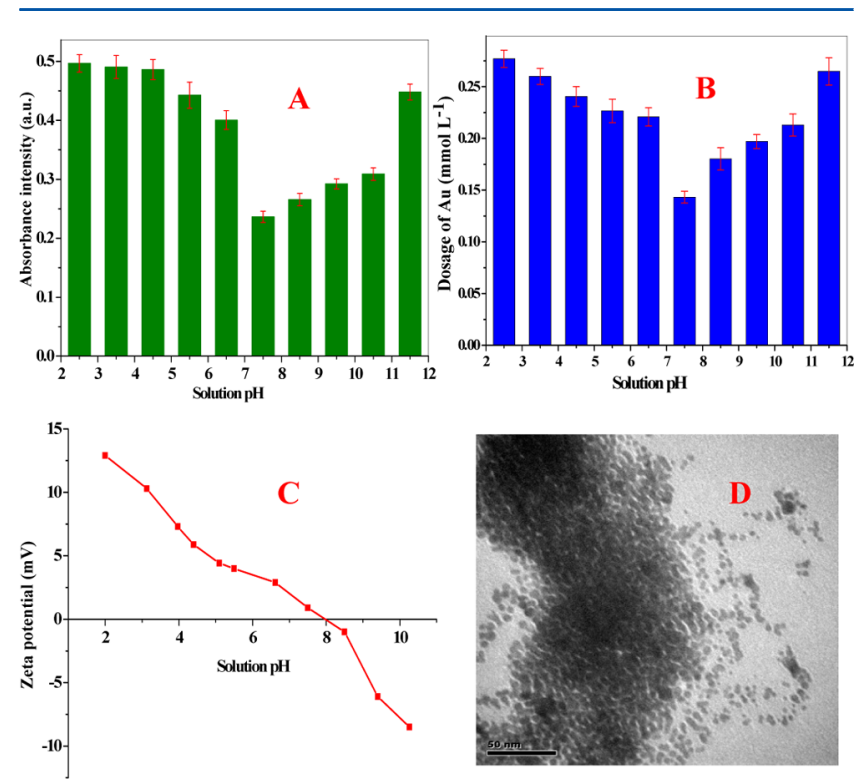

Figure 5. Absorbance (A), concentration of Au element (B), and $\zeta$-potential (C) of Au@PEG-F NPs suspension at different pHs, and TEM image of Au@PEG-F NPs (D) at pH 7.5.

$\mathrm{pH}$ was consistent with the variation of the corresponding $\mathrm{Au}$ element amount (Figure 5B) at different solution pHs. To interpret the effect of solution pH on Au@PEG-F NPs stability, we measured $\zeta$-potentials of Au@PEG-F NPs in aqueous solutions. The isoelectric point (IEP) of the Au NPs sensor was found at about $\mathrm{pH} 8.0$ (Figure $5 \mathrm{C}$ ). At $\mathrm{pH}<\mathrm{pH}_{\mathrm{IEP}}$, the oxygen atoms in the $\mathrm{PEG}$ chains can adsorb $\mathrm{H}_{3} \mathrm{O}^{+}$through hydrogen bonds, leading to the positive surface of Au@PEG-F NPs, while the $\mathrm{OH}$ groups of PEG-thiols can ionize to form negatively charged surface at $\mathrm{pH}>\mathrm{pH}_{\mathrm{IEP}}$. Therefore, the PEG chains maintain a brushlike conformation in which the chains extend out into solution both in acid and strong alkaline solution due to repulsive reaction between positively or negatively charged PEG-thiols, imparting the stability of Au@PEG-F NPs colloids. At about $\mathrm{pH}_{\mathrm{IEP}}$, the electrostatic interaction between PEG chains decreases and cannot counteract the hydrophobic interaction among the hydrophobic regions $\left(-\mathrm{CH}_{2}-\mathrm{CH}_{2}-\right)$, and the PEG chains display a conformation as a random coil, allowing the F-thiols exposure in solution and inducing the 

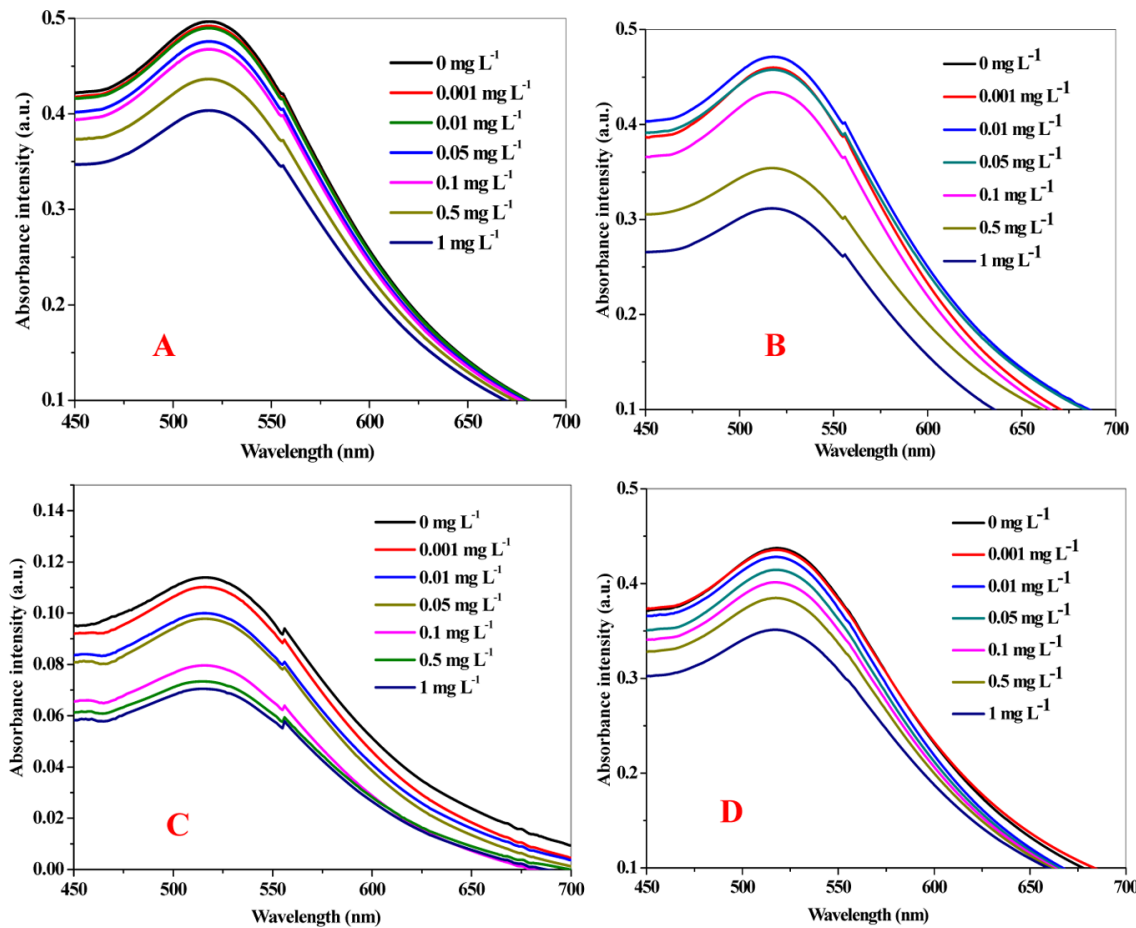

Figure 6. UV-vis spectra of the Au@PEG-F NPs in response to different concentrations of PFOS at pH 2.5 (A), pH 4.5 (B), pH 7.5 (C), and $\mathrm{pH} 11.5$ (D), from lower to upper: 0, 1, 10, 50, 100, 500, and $1000 \mu \mathrm{g} \mathrm{L}{ }^{-1}$ of PFOS.

interaction among Au NPs. This could be confirmed by their TEM images, in which highly aggregated Au@PEG-F NPs were observed at $\mathrm{pH} 7.5$ (Figure 5D). In literature, PEG-modified $\mathrm{Au}$ NPs are stable at wide $\mathrm{pH}$ range due to the strong interactions between water and PEG chains. But the molecular weight of these PEGs is usually larger than $1000 \mathrm{Da}^{41,42}$ In this study, the molar mass of PEG units was low $(M=281)$ and the interactions between water and PEG chains were weak, so the stability of Au@PEG-F NPs was sensitive to solution pH.

The sensitivity of the assay to PFCs at different solution $\mathrm{pHs}$ was studied with the concentrations of PFOS ranging in $1-1000 \mu \mathrm{g} \mathrm{L}^{-1}$. In acid and strong alkaline solution, F-thiols on Au NPs sensors were sheltered by the long-chain PEG-thiols in brushlike conformation, which impeded the interaction between PFCs and F-thiols. Therefore, the sensitivities of the assay to PFCs at $\mathrm{pH} 2.5$ and $\mathrm{pH} 11.5$ were low (Figure 6, parts $\mathrm{A}$ and $\mathrm{D}$ ). In neutral and weak alkaline solution, the F-thiols were exposed to solution and readily contacted with PFCs. The sensitivities of the assay should be high in these $\mathrm{pH}$ ranges. However, we found that the assay showed low sensitivity to PFOS at $\mathrm{pH} 7.5-9.5$ perhaps due to the extremely low amount of $\mathrm{Au}$ NPs sensors that remained in the reaction solution (Figure 6C). In the $\mathrm{pH}$ range of 3.5-6.5, the sensitivities of the assay to PFOS were higher and generally increased with $\mathrm{pH}$ value (Figures $1 \mathrm{~B}$ and $6 \mathrm{~B}$ ). But the highest sensitivity to PFOS was achieved at $\mathrm{pH} 5.5$; thus, solution $\mathrm{pH}$ of the assay was set at about $\mathrm{pH} 5.5$ in the following experiment.

Colloidal Au NPs have a tendency to easily aggregate in the presence of high salts, which is the primary obstacle for the application of most colorimetric assays in environmental samples. $^{12}$ In this study, we studied the effects of salts (including $\mathrm{NaCl}, \mathrm{MgCl}_{2}$, and $\mathrm{CaCl}_{2}$ ) concentrations on the stability of Au@PEG-F NPs. The concentration of these salts ranged in $0.5-500 \mathrm{mM}$. We found that the Au@PEG-F NPs colloid remained very stable in the whole salts concentration range. The absorption intensities of reaction solution at $520 \mathrm{~nm}$ were nearly unchanged compared with the controlled solution (Figure 7). In the presence of $500 \mathrm{mM}$ of each salt, $500 \mu \mathrm{g} \mathrm{L}^{-1}$

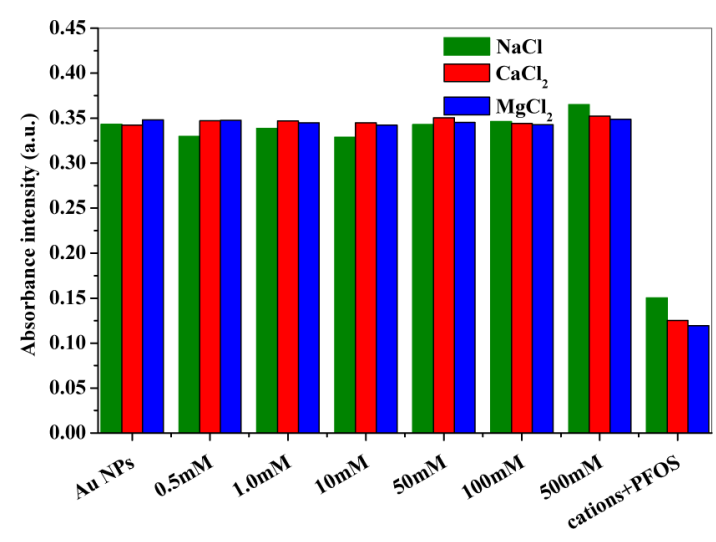

Figure 7. Absorbance of Au@PEG-F NPs suspension in the presence of different concentrations of $\mathrm{NaCl}, \mathrm{MgCl}_{2}$, and $\mathrm{CaCl}_{2}$ with and without PFOS $\left(500 \mu \mathrm{g} \mathrm{L}^{-1}\right)$; the amount of each salt in solution containing PFOS is $500 \mathrm{mM}$.

of PFOS was added to the reaction solution, and the intensities at $520 \mathrm{~nm}$ decreased obviously, indicating that the assay was not affected by the coexisting high amount of salts. The extremely high stability of PEG-SH-modified Au NPs colloid in salt solution was also reported by Gao et al. ${ }^{43}$ They suggested that the PEG polymers can provide steric hindrance among Au NPs and result in an increased stability of Au NPs in solution. Although the molecular weight of PEG-SH in this study was far lower than the one at Gao's study $(5 \mathrm{kDa})$, the Au@PEG-F NPs still showed excellent anti-interference ability to high concentration of salts, indicating the application potential of this assay in environmental samples. 
Selectivity. The selectivity of the assay was investigated by addition of nonfluorinated alkyl carboxyl acid, alkyl alcohol, alkylamine, and cationic and anionic surfactants bearing long carbon chains to the reaction solution including octanoic acid, decanoic acid, dodecanoic acid, 1,2-dodecandiol, 1-dodecylamine, 1-hexadecylamine, sodium dodecyl sulfate (SDS), sodium hexadecanesulfonate (SHDS), sodium octadecanesulfonate (SODS), sodium dodecyl benzenesulfonate (SDBS), and hexadecyltrimethylammonium bromide (CTAB). In the presence of $500 \mu \mathrm{g} \mathrm{L}^{-1}$ of each nonfluorinated compound, the absorbance of reaction solution did not change, indicating that the assay was insensitive to these nonfluorinated compounds (Figure 8). We further tested the selectivity of

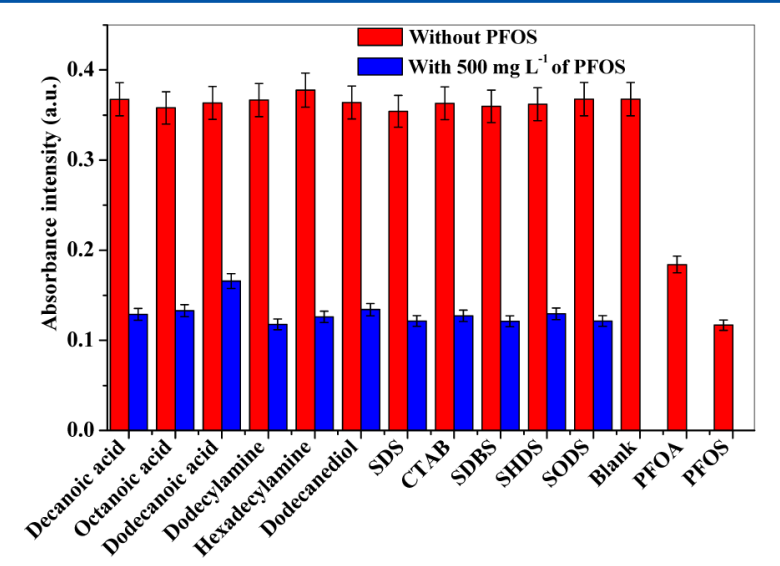

Figure 8. Absorbance of Au@PEG-F NPs suspension in the presence $500 \mu \mathrm{g} \mathrm{L}^{-1}$ of each nonfluorinated compound with and without $500 \mu \mathrm{g} \mathrm{L}^{-1}$ of PFOS.

the assay to PFCs by recording the UV-vis spectrum of $\mathrm{Au}$ NPs suspension with the coexistence of PFOS and each of the nonfluorinated compounds $\left(500 \mu \mathrm{g} \mathrm{L}^{-1}\right)$. With the addition of $500 \mu \mathrm{g} \mathrm{L}^{-1}$ PFOS, the peak intensity at $520 \mathrm{~nm}$ decreased remarkably and was comparable to that without nonfluorinated compounds. These results disclosed that the assay had excellent selectivity to PFCs over the nonfluorinated compounds.

Detection Sensitivity to Different PFCs. Different amounts of each perfluorinated compound were added to the reaction solution. The final concentrations for short-chain PFBS (F4), PFHxS (F6), and PFHpA (F6) ranged in 0.1$10000 \mu \mathrm{g} \mathrm{L}^{-1}$; those for long-chain PFCs (F7-F17) were in the range of $0.1-1000 \mu \mathrm{g} \mathrm{L}^{-1}$. The UV-vis spectrum of each reaction solution under the optimal condition was recorded.
The dose-dependent responses of PFCs are shown in Table 2. In a wide concentration range, the UV-vis absorbance decreased linearly with PFCs concentration. The sensitivities of the assay to PFCs increased with the elongation of perfluoroalkyl chain $\left(\mathrm{CF}_{2}\right)$. For PFBS, obvious dose-dependent response to the assay only could be discerned when the PFBS concentration was higher than $1000 \mu \mathrm{g} \mathrm{L}^{-1}$. As to $\mathrm{PFHxS}$ and PFHpA, the response of the two PFCs to the assay was identified when their concentrations were higher than $100 \mu \mathrm{g} \mathrm{L}^{-1}$. The hydrophobicity of PFCs increased with the elongation of $\mathrm{CF}_{2}$; therefore, $\mathrm{Au}$ NPs probes adsorbed with longer-chain PFCs were more readily precipitated from water solution, which resulted in low sensitivity of this assay to short-chain PFCs. For long-chain PFCs (perfluoroalkyl chain $\mathrm{CF}_{2} \geq 7$ ), the decrease of absorbance could be observed even when the concentration was as low as $10 \mu \mathrm{g} \mathrm{L}^{-1}(11-24 \mathrm{nM})$. The slopes of the linear equations (representing the sensitivity of the assay to PFCs) for three perfluorinated sulfonates (PFSAs) and seven perfluorinated carboxylic acids (PFCAs) increased generally with the elongation of $\mathrm{CF}_{2}$. This trend was more obvious if we normalized the linear equations with molecular weight. Table 2 also exhibits that the assay demonstrates higher sensitivity to perfluorinated sulfonates than perfluorinated carboxylic acids with the same length of perfluoroalkyl chain, which might be caused by the higher hydrophobicity of PFSAs than those of PFCAs.

In general, the colorimetric assays based on Au@PEG-F NPs showed high sensitivities for long-chain PFCs $\left(\mathrm{CF}_{2} \geq 7\right)$ with wide linear range. The concentrations of total PFCs in natural water samples are in the range of tenths to hundreds of parts per trillion. ${ }^{1-11}$ Only application of LC/MS/MS allows better limits of detection (LODs) to be obtained. However, the drawback of the mass spectrometer is its instrumental price and the running cost. There are also challenges with LC/MS/MS systems that are composed of fluorinated polymers from which PFCs, most notably PFOA, can be leached, causing background problems. ${ }^{8}$ The LODs obtained for derivatization techniques coupled with spectrofluorometric detector, ${ }^{6}$ HPLC-FLD, ${ }^{7}$ $\mathrm{GC}-\mathrm{ECD},{ }^{8,9}$ and GC-EI-MS/MS ${ }^{8,9}$ were in the range of tens of parts per billion to parts per million levels. The LODs for the PFCs with ion chromatography coupled with conductivity detection ${ }^{10}$ and the electrophoretic method ${ }^{11}$ were around the parts per million level and too high for their application to directly analyze PFCs in environmental samples (excluding cases of heavy contamination). Water samples are required to be pretreated with solid-phase extraction (SPE) or liquidliquid separation methods in advance. Even with the LC/MS/MS

Table 2. Linear Range, Linear Equation, and Correlation Coefficients of the Colorimetric Assay to Different PFCs

\begin{tabular}{|c|c|c|c|c|c|}
\hline \multirow[b]{2}{*}{ PFCs } & \multicolumn{2}{|c|}{ linear range } & \multicolumn{2}{|c|}{ linear equation } & \multirow[b]{2}{*}{$R^{2}$} \\
\hline & $\mathrm{mg} \mathrm{L}^{-1}$ & $\mu \mathrm{mol} \mathrm{L}-1$ & $\mathrm{mg} \mathrm{L}^{-1}$ & $\mu \mathrm{mol} \mathrm{L}-1$ & \\
\hline PFBS & $1.0-10$ & $3.3-33$ & $y=-0.009 x+0.181$ & $y=-0.003 x+0.181$ & 0.982 \\
\hline PFHxS & $0.05-5.0$ & $0.125-12.5$ & $y=-0.055 x+0.387$ & $y=-0.022 x+0.387$ & 0.990 \\
\hline PFOS & $0.01-1.0$ & $0.02-2.0$ & $y=-0.301 x+0.411$ & $y=-0.151 x+0.411$ & 0.995 \\
\hline PFHpA & $0.05-5.0$ & $0.137-13.8$ & $y=-0.030 x+0.248$ & $y=-0.011 x+0.248$ & 0.995 \\
\hline PFOA & $0.01-1.0$ & $0.024-2.4$ & $y=-0.173 x+0.355$ & $y=-0.072 x+0.355$ & 0.994 \\
\hline PFNA & $0.01-1.0$ & $0.021-2.1$ & $y=-0.147 x+0.245$ & $y=-0.068 x+0.245$ & 0.993 \\
\hline PFTrDA & $0.01-1.0$ & $0.015-1.5$ & $y=-0.278 x+0.395$ & $y=-0.171 x+0.395$ & 0.993 \\
\hline PFTeDA & $0.01-1.0$ & $0.014-1.4$ & $y=-0.276 x+0.396$ & $y=-0.194 x+0.396$ & 0.996 \\
\hline PFHxDA & $0.01-1.0$ & $0.013-1.2$ & $y=-0.257 x+0.392$ & $y=-0.209 x+0.392$ & 0.997 \\
\hline PFODA & $0.01-1.0$ & $0.011-1.1$ & $y=-0.12 x+0.398$ & $y=-0.11 x+0.398$ & 0.992 \\
\hline
\end{tabular}


method, PFCs always need to be preconcentrated with the SPE method from water samples. After preconcentration for hundreds of times, the sensitivities of these methods developed for PFCs will increase greatly and the LODs can decrease to sub parts per trillion to parts per billion levels, which meets the requirements of high sensitivity and selectivity for PFCs measurement. In the designed concentration range, the response of the colorimetric assay based on the Au@PEG-F NPs sensor we developed to long-chain PFCs $\left(\mathrm{CF}_{2} \geq 7\right)$ was observed even as the concentration of these PFCs was as low as $10 \mu \mathrm{g} \mathrm{L}^{-1}$. The sensitivity of this assay to PFCs was superior to or comparable with those of HPLC-FLD and GC-ECD techniques, indicating that this colorimetric assay could be employed to measure total PFCs in real water samples after preconcentrating with an SPE method. In addition, the developed assay was lower in cost with use of much simpler and more commonly employed instruments. Both the synthesis of Au@PEG-F NPs probers and implementation of the assay were very simple and convenient because we did not need to derivatize PFCs and add any additives such as organic dyes and enzymes into the reaction solution.

The Au@PEG-F NPs sensor was employed to analyze PFOS in tap water and river water samples. Water samples were filtered through a nylon film $(0.45 \mu \mathrm{m})$ before extraction. An aliquot of $200 \mathrm{~mL}$ of blank water samples and those spiked with $10 \mathrm{ng}$ of PFOS were extracted with an HLB cartridge $(6 \mathrm{cc}$, 150 mg; Waters Corp. Milford, U.S.A.), respectively. After sample loading, the cartridges were washed with $5 \mathrm{~mL}$ of purified water and dried with a gentle steam of $\mathrm{N}_{2}$. The adsorbed PFOS was eluted with $5 \mathrm{~mL}$ of methanol. The eluate was then concentrated to $0.2 \mathrm{~mL}$ and added to $0.8 \mathrm{~mL}$ of $\mathrm{Au}$ NPs solution. After reaction for $30 \mathrm{~min}$, the $\mathrm{Au}$ NPs reaction solution was centrifuged and the supernatant was measured using UV-vis spectrometry at $520 \mathrm{~nm}$. The result showed that no PFOS was detected in all the water samples. The recoveries of PFOS in the river water and tap water samples were $85 \% \pm$ $10 \%$ and $115 \% \pm 12 \%$, respectively.

\section{CONCLUSIONS}

In summary, we have demonstrated the feasibility of using $\mathrm{Au}$ NPs based colorimetric assay in the detection of PFCs in aqueous solution. The preparation of Au NPs probe modified with mixed perfluoroalkyl and PEG groups can be achieved just by soaking Au NPs in ethanol solvent containing thiols. The strong and specific F-F interaction between PFCs and F-thiols results in the adsorption of PFCs on Au NPs, which increases the hydrophobicity of the Au NPs and leads to the participation of the Au NPs sensors. The color of the reaction solution and UV-vis absorbance changes with PFCs concentration. This developed assay is lower in cost and more convenient to implement and shows excellent selectivity and sensitivities to PFCs. To our knowledge, this is the first application of the specific fluorous-fluorous interactions and $\mathrm{Au}$ NPs based probes for colorimetric assays for PFCs or even emerging pollutants. More importantly, this study opens new opportunities for the design of novel sensing strategies for organic environmental pollutants.

\section{AUTHOR INFORMATION}

\section{Corresponding Author}

*Phone: +010 62849239. Fax: +010 62849182. E-mail: caiyaqi@rcees.ac.cn.

\section{Notes}

The authors declare no competing financial interest.

\section{ACKNOWLEDGMENTS}

This work was jointly supported by the National Basic Research Program of China (2011CB936001) and National Natural Science Foundation of China (21277002, 21277152, 21321004).

\section{REFERENCES}

(1) Pan, Y. Y.; Shi, Y. L.; Wang, J. M.; Cai, Y. Q. Sci. China: Chem. 2011, 54, 552-558.

(2) Shi, Y. L.; Pan, Y. Y.; Yang, R. Q.; Wang, Y. W.; Cai, Y. Q. Environ. Int. 2010, 36, 46-50.

(3) Tomy, G. T.; Budakowski, W.; Halldorson, T.; Helm, P. A.; Stern, G. A.; Friesen, K.; Pepper, K.; Tittlemier, S. A.; Fisk, A. T. Environ. Sci. Technol. 2004, 38, 6475-6481.

(4) Martin, J. W.; Smithwick, M. M.; Braune, M. B.; Hoekstra, P. F.; Muir, D. C. G.; Mabury, S. A. Environ. Sci. Technol. 2004, 38, 373-380.

(5) Moody, C. A.; Kwan, W. C.; Martin, J. W.; Muir, D. C. G.; Mabury, S. A. Anal. Chem. 2001, 73, 2200-2206.

(6) Takayanagi, T.; Yamashita, H.; Motomizu, S.; Musijowski, J.; Trojanowicz, M. Talanta 2008, 74, 1224-1230.

(7) Poboży, E.; Król, E.; Wójcik, L.; Wachowicz, M.; Trojanowicz, M. Microchim. Acta 2011, 172, 409-417.

(8) Scott, B. F.; Moody, C. A.; Spencer, C.; Small, J. M.; Muir, D. C. G.; Mabury, S. A. Environ. Sci. Technol. 2006, 40, 6405-6410.

(9) Dufková, V.; Čabala, R.; Maradová, D.; Štícha, M. J. Chromatogr., A 2009, 1216, 8659-8664.

(10) Sanderson, H.; Boudreau, T. M.; Mabury, S. A.; Cheong, W. J.; Solomon, K. R. Environ. Toxicol. Chem. 2002, 21, 1490-1496.

(11) Wojcik, L.; Szostek, B.; Maruszak, W.; Trojanowicz, M. Electrophoresis 2005, 26, 1080-1088.

(12) Liu, D. B.; Wang, Z.; Jiang, X. Y. Nanoscale 2011, 3, 1421-1433.

(13) Elghania, R.; Storhoff, J. J.; Mucic, R. C.; Letsinger, R. L.; Mirkin, C. A. Science 1997, 277, 1078-1081.

(14) Jang, H. J.; Kim, Y. K.; Ryoo, S. R.; Kim, M. H.; Min, D. H. Chem. Commun. 2010, 46, 583-585.

(15) Xia, F.; Zuo, X. L.; Yang, R. Q.; Xiao, Y.; Kang, D.; ValléeBélisle, A.; Gong, X.; Yuen, J. D.; Hsu, B. B. Y.; Heegera, A. J.; Plaxco, K. W. Proc. Natl. Acad. Sci. U.S.A. 2010, 107, 10837-10841.

(16) Saha, K.; Agasti, S. S.; Kim, C.; Li, X. N.; Rotello, V. M. Chem. Rev. 2012, 112, 2739-2779.

(17) Xue, X. J.; Wang, F.; Liu, X. G. J. Am. Chem. Soc. 2008, 130, 3244-3245.

(18) Zhuang, X. M.; Wang, D. L.; Wang, D. L.; Yang, L. F.; Yu, P.; Jiang, W.; Mao, L. Q. Analyst 2013, 138, 3046-3052.

(19) Chen, Z.; Luo, S. L.; Liu, C. B.; Cai, Q. Y. Anal. Bioanal. Chem. 2009, 395, 489-494.

(20) Deng, J. J.; Yu, P.; Yang, L. F.; Mao, L. Q. Anal. Chem. 2013, 85, 2516-2522.

(21) Qian, Q.; Deng, J. J.; Wang, D. L.; Yang, L. F.; Yu, P.; Mao, L. Q. Anal. Chem. 2012, 84, 9579-9584.

(22) Ai, K. L.; Liu, Y. L.; Lu, L. H. J. Am. Chem. Soc. 2009, 131, 9496-9497.

(23) Zhang, J.; Wang, L. H.; Pan, D.; Song, S. P.; Boey, F. Y. C.; Zhang, H.; Fan, C. H. Small 2008, 4, 1196-1200.

(24) Wang, J.; Wang, L. H.; Liu, X. F.; Liang, Z. Q.; Song, S. P.; Li, W. X.; Li, G. X.; Fan, C. H. Adv. Mater. 2007, 19, 3943-3946.

(25) Jiang, Y.; Zhao, H.; Lin, Y. Q.; Zhu, N. N.; Ma, Y. R.; Mao, L. Q. Angew. Chem., Int. Ed. 2010, 49, 4800-4804.

(26) Zhang, Y. F.; Li, B. X.; Chen, X. L. Microchim. Acta 2009, 168, 107-113.

(27) Dasary, S. S. R.; Senapati, D.; Singh, A. K.; Anjaneyulu, Y.; Yu, H. T.; Ray, P. C. ACS Appl. Mater. Interfaces 2010, 2, 3455-3460.

(28) Jiang, Y.; Zhao, H.; Zhu, N. N.; Lin, Y. Q.; Yu, P.; Mao, L. Q. Angew. Chem., Int. Ed. 2008, 47, 8601-8604.

(29) Liu, D. B.; Chen, W. W.; Wei, J. H.; Li, X. B.; Wang, Z.; Jiang, X. Y. Anal. Chem. 2012, 84, 4185-4191. 
(30) Zhang, M.; Liu, Y. Q.; Ye, B. C. Chem. Commun. 2011, 47, 11849-11851.

(31) de Wolf, E.; van Koten, G.; Deelman, B.-J. Chem. Soc. Rev. 1999, $28,37-41$.

(32) Matsugi, M.; Curran, D. P. Org. Lett. 2004, 16, 2717-2720.

(33) Daley, A. B.; Oleschuk, R. D. J.Chromatogr., A 2009, 1216, 772780.

(34) Daley, A. B.; Xu, Z. P.; Oleschuk, R. D. Anal. Chem. 2011, 83, $1688-1695$.

(35) Glatz, H.; Blay, C.; Engelhardt, H.; Bannwarth, W. Chromatographia 2004, 59, 567-570.

(36) Pearson, W. H.; Berry, D. A.; Stoy, P.; Jung, K. Y. J. Org. Chem. 2005, 70, 7114-7122.

(37) Brittain, S. M.; Ficarro, S. B.; Brock, A.; Peters, E. C. Nat. Biotechnol. 2005, 23, 463-468.

(38) Dass, A.; Guo, R.; Tracy, J. B.; Balasubramanian, R. Langmuir 2008, 24, 310-315.

(39) Yonezawa, T.; Onoue, S. Y.; Kimizuk, N. Langmuir 2001, 17, 2291-2293.

(40) Gentilini, C.; Evangelista, F.; Rudolf, P.; Franchi, P.; Lucarini, M.; Pasquato, L. J. Am. Chem. Soc. 2008, 130, 15678-15682.

(41) Huang, L.; Nishinari, K. J. Polym. Sci., Part B: Polym. Phys. 2001, 39, 496-506.

(42) Liu, Y. L.; Shipton, M. K.; Ryan, J.; Kaufman, E. D.; Franzen, S.; Feldheim, D. L. Anal. Chem. 2007, 79, 2221-2229.

(43) Gao, J.; Huang, X. Y.; Liu, H.; Zan, F.; Re, J. C. Langmuir 2012, $28,4464-4471$. 